\title{
The Contributions of Visual Flow and Locomotor Cues to Walked Distance Estimation in a Virtual Environment ${ }^{* \dagger}$
}

\author{
J. L. Campos, J. S. Butler, B. J. Mohler, \& H. H. Bülthoff \\ Max Planck Institute for Biological Cybernetics
}

\section{Introduction}

Traversed distance perception involves estimating the extent of selfmotion as one travels from one position in space to another. As such, it is a multi-modal experience in which information from both visual flow and locomotor cues (i.e. proprioceptive, efference copy and vestibular cues) jointly specify the magnitude of self-motion. While recent evidence has demonstrated the extent to which each of these cues can be used independently to estimate traversed distance, relatively little is known about how they are integrated when simultaneously present. Evaluating multi-modal cue integration in the context of dynamic locomotor behaviour is important to both understanding issues related to self-motion perception, as well as perceptual-motor coupling in real and virtual environments.

\section{Methods}

In this task, 8 participants experienced a travelled distance by moving down a richly textured, "infinitely" long virtual hallway viewed through a head-mounted display (HMD), until reaching an unmarked, predefined distance $(4,6,8$ or $10 \mathrm{~m})$ at which point the screen went blank (Figure 1). Subsequently, they turned 180 degrees, the hallway reappeared and a target object (striped sphere) was presented $2 \mathrm{~m}$ in front of them. Participants were then required to adjust the target until the in-depth, self-to-target distance matched the distance they had previously travelled.
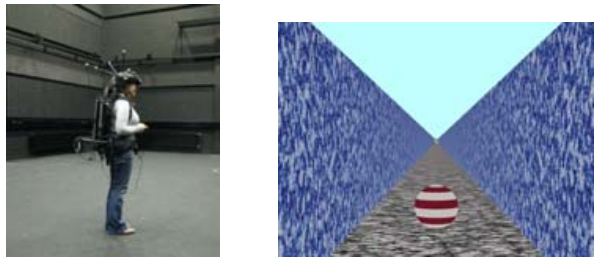

Figure 1: Left: The experiment took place in a large, fully tracked, free-walking space, $12 \times 15$ meters in size. Participants' positions were tracked using an optical tracking system (16 Vicon Mx13 cameras). Right: A HMD (eMagin, Z800; 40 deg. diagonal FOV) was used to present the virtual environment.

Four conditions were conducted and all participants completed each condition. All trials within a condition were blocked.

-Visual-Locomotor Congruent (VLCon): The relation between the motorically-specified distance through walking and the distance specified by visual flow was constant.

- Visual-Locomotor Incongruent (VLIncon): The relation between the visual flow and the motorically-specified distance during walking was varied by manipulating the visual gain on each trial. Gain values of $0.7,1.0$ (same as VLCon) and 1.4 were used.

*Correspondence: jenny.campos@tuebingen.mpg.de

$\dagger$ Many thanks to Michael Weyel for his invaluable programming and technical assistance and to Dr. Hong Jin Sun for many helpful comments.

Copyright $(0) 2007$ by the Association for Computing Machinery, Inc.

Permission to make digital or hard copies of part or all of this work for personal or classroom use is granted without fee provided that copies are not made or distributed for commercial advantage and that copies bear this notice and the full citation on the first page. Copyrights for components of this work owned by others than ACM must be honored. Abstracting with credit is permitted. To copy otherwise, to republish, to post on servers, or to redistribute to lists, requires prior specific permission and/or a fee. Request permissions from Permissions Dept, ACM Inc., fax +1 (212) $869-0481$ or e-mail permissions@acm.org.

APGV 2007, Tübingen, Germany, July 26-27, 2007.

(C) 2007 ACM 978-1-59593-670-7/07/0007 $\$ 5.00$
- Vision Alone (VisAlone): Participants stood stationary while viewing a visual trajectory of the distance travelled through the hallway. The speed of the visual information was matched to the participant's own walking trajectories in the VLCon condition (0.7 and 1.4 magnitudes were also included).

- Locomotor Alone (LocAlone): Participants walked in the complete absence of vision (black screen).

\section{Results and Discussion}

When distances were travelled with VisAlone they were estimated to be significantly shorter than when travelled with LocAlone (Fig. 2A). In the VLCon condition, participants' distance estimates fell between the VisAlone and the LocAlone conditions. These results confirm and expand upon previous findings in real world traversed distance estimation tasks in which subjects have been shown to judge travelled distance intervals to be shorter when walking with vision than when walking without vision (Sun et al., 2004; Campos et al., submitted). When the two cues were combined in the VLCon condition, estimates more closely approximated the estimates for the LocAlone condition than the VisAlone condition. This tendency towards locomotor cues was particularly evident at longer distances.
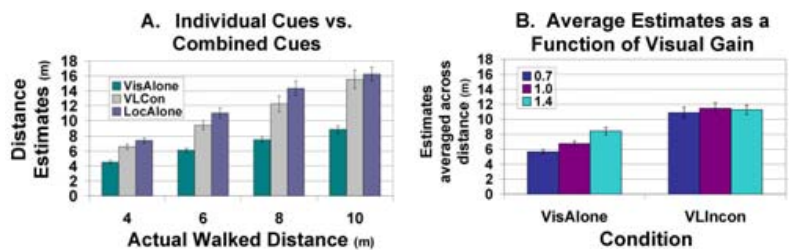

Figure 2: A: Average distance estimates for the VLCon, VisAlone and LocAlone conditions. B: Estimates averaged across the four distances as a function of visual gain/magnitude.

As shown in Fig.2B, participants were sensitive to the changes in the magnitude of visual flow in the VisAlone condition, such that their distance estimates were shorter for lower gains and longer for higher gains. However, when a similar visual manipulation occurred during trials in which locomotor cues were also available (VLIncon), the effect of the visual gain was no longer observed. These results may reflect a decrease in the perceived reliability of visual cues in the VLIncon task. In the VLCon condition we see that the mere presence of visual information changed responding compared to LocoAlone and yet in the VLIncon condition there appeared to be no effect of visual gain. The different pattern of cueweighting observed in the VLCon and VLIncon conditions may relate to a sensitivity of the statistical stability of multimodal cue relations over trials and highlights the dynamic nature of sensory integration.

\section{References}

Campos, J. L., Byrne, P., And Sun, H.-J. Quantifying the effects of optic flow on the estimation of walked distances. submitted.

Sun, H.-J., Campos, J. L., Young, M. E., and Chan, G. S. W. 2004. The contributions of static visual cues, nonvisual cues, and optic flow in distance estimation. Perception 33, 4569. 